Revista Electrónica Teoría de la Educación.

Educación y Cultura en la Sociedad de la Información.

http://www.usal.es/teoriaeducacion

Vol. 7. No2. Diciembre 2006

\title{
ESTIMULACIÓN EMOCIONAL DE LOS VIDEOJUEGOS: EFECTOS EN EL APRENDIZAJE
}

La industria de los videojuegos se encuentra en amplia expansión, ha trascendido el área de la diversión propiamente dicha para abordar el campo educativo/formativo en forma explícita. El objetivo de este trabajo es destacar la capacidad que tienen los videojuegos para potenciar el aprendizaje debido a su capacidad inmersita. La inmersión implica involucrar emocionalmente al videojugador en la situación de juego. Esta implicación de los aspectos emocionales son determinantes para el aprendizaje y hay razones neurofisiológicas que respaldan estos hechos explicados mediante el modelo de Cerebro Triuno. Los resultados de esta investigación analítica-documental confirman el papel de las emociones para estimular el aprendizaje y como este es potenciado por las características emocionales de los videojuegos.

Palabras clave: cerebro triuno y aprendizaje, emociones en el juego, videojuegos

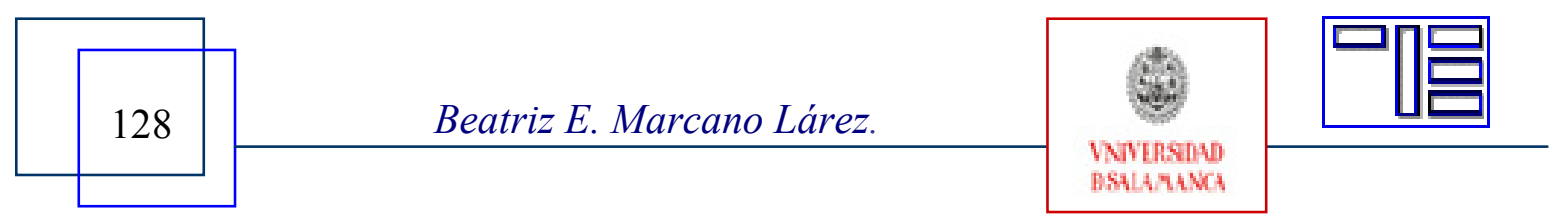




\section{EMOTIONAL STIMULATION OF VIDEO GAMES: EFFECTS IN THE LEARNING PROCESSES}

The industry of the video games is in wide expansion. It has transcended the area of amusement to move towards the educational field. The objective of this work is to highlight the capability video games have in the fostering of learning processes. Video games have the capacity of immersion that allows video players to become emotionally involved in a specific game situation. The implication of the emotional aspects is significant for the learning process and there are neurophysiologic reasons that support these facts based on the pattern of Triuno brain. The results of this analytic-documental research confirm the important role of emotions in stimulating learning as well as the positive influence reached by the emotional characteristics of the video games.

Key words: Triuno brain and learning, emotions, video games.

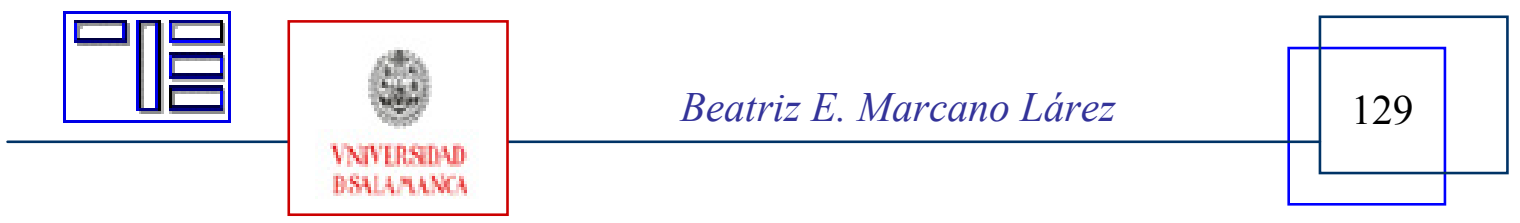


Revista Electrónica Teoría de la Educación.

Educación y Cultura en la Sociedad de la Información.

http://www.usal.es/teoriaeducacion

Vol. 7. N2. Diciembre 2006

\section{STIMULATION EMOTIONNELLE DES JEUX VIDÉO : EFFETS L'APPREN- TISSAGE}

L'industrie de jeux vidéo est dans une vaste expansion. Elle a dépassé le secteur de l'amusement pour aborder le domaine du champ éducatif explicitement. L'objectif de ce travail est de souligner la capacité que ceux jeux ont dans le renforcement des apprentissages. Les jeux vidéo ont la capacité d'immersion qui permet aux joueurs de devenir avec émotion dans une situation spécifique de jeu. L'implication des aspects émotifs est significative pour l'apprentissage et il y a des raisons neurophysiologiques qui soutiennent ces faits basés sur le modèle du cerveau de Triuno. Les résultats de cette recherche analytique -documentaire confirment le rôle important des émotions dans la stimulation $\mathrm{du}$ apprentissage aussi bien comme l'influence positive q'il a par les caractéristiques émotives des jeux vidéo.

Mots clefs: cerveau de Triuno et apprentissages, émotions, jeux vidéo.

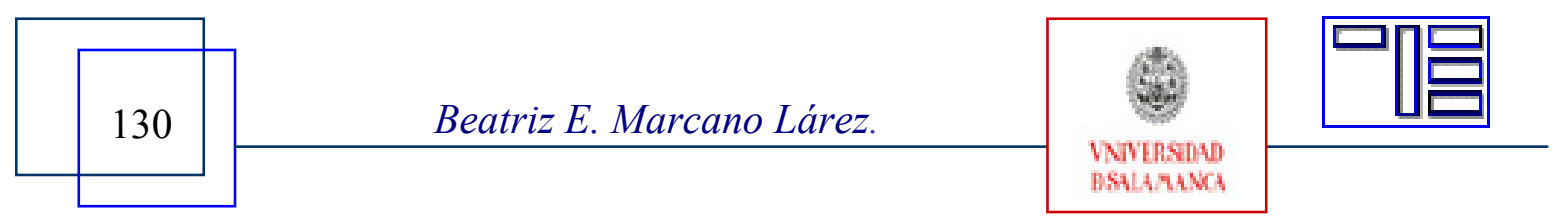




\section{ESTIMULACIÓN EMOCIONAL DE LOS VIDEOJUEGOS: EFECTOS EN EL APRENDIZAJE}

Beatriz E. Marcano Lárez

beatriz_marcano@yahoo.com

Universidad de Oriente- Nueva Esparta. Programa de Educación Integral (VENEZUELA)

\section{1.- INTRODUCCIÓN}

El desarrollo tecnológico y las nuevas herramientas de creación han permitido generar juegos electrónicos también llamados softwares de entretenimiento o videojuegos con características capaces de "sumergir" al videojugador en entornos virtuales de juego, que favorecen la generación de emociones y a su vez estimulan el aprendizaje. Teóricamente esta relación entre emociones y aprendizajes se apoya en los aportes de las neurociencias y específicamente en el modelo de Cerebro Triuno, según el cual los aprendizajes se aceleran con la multiestimualción sensorial y la emocionalidad. El diseño de los videojuegos permite cumplir con estas condiciones, de allí que se quieran exponer algunos aprendizajes estimulados con el uso de los videojuegos.

\section{2.- ESTIMULACIÓN EMOCIONAL DE LOS VIDEOJUEGOS}

Los videojuegos son softwares multimedia diseñados para entretener. En sus inicios estas creaciones eran realizadas a manera de diversión y de reto para los programadores que se dedicaban a ello, sin embargo al probar estos software de entretenimientos con el público y observar el gran potencial de lucro que tenían esta actividad de creación pasó a ser realizada por grupos de especialistas en diferentes áreas (programación, diseño gráfico, arte, ingenieros de sonido, etc.), buscando que el producto resultara cada vez más excitante para los potenciales usuarios. De allí que la creación y diseño de los videojuegos a medida que ha pasado el tiempo, pretenda penetrar más en la psicología y en la mente de las masas. Es por eso que cada vez se generan más investigaciones y estudios en el área, para lograr comprender de qué manera un juego le resulta más atractivo a un usuario. Por supuesto, ésta atractibilidad de los juegos va a depender de los

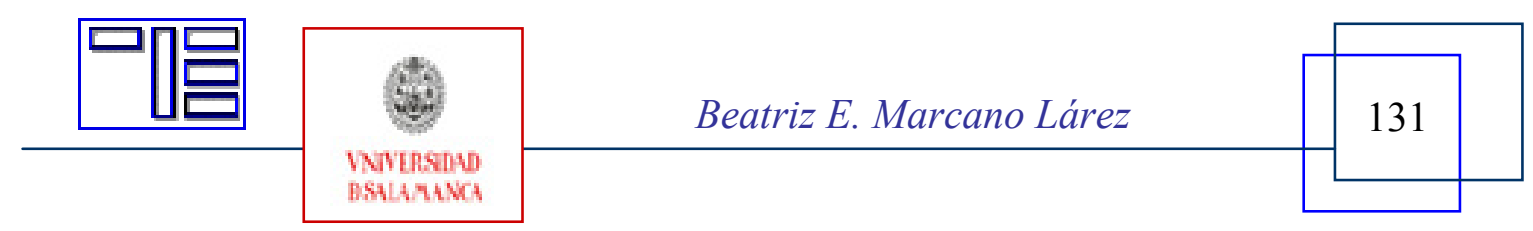


Revista Electrónica Teoría de la Educación.

Educación y Cultura en la Sociedad de la Información.

http://www.usal.es/teoriaeducacion

Vol. 7. No2. Diciembre 2006

diferentes tipos de usuarios, por la influencia que tienen los factores personales e individuales en los gustos; sin embargo, hay factores motivacionales generales que hacen a los videojuegos atractivos. Estos últimos factores, en muchos casos son compartidos por los juegos no electrónicos. Entre estos factores de atractibilidad, se encuentran: la posibilidad de competición, el reto, la posibilidad de interactuar, las acciones que permite hacer y las emociones que permita vivir.

Otro elemento importante de atractibilidad de los videojuegos es el relacionado con los aspectos gráficos y el diseño, es decir, todos aquellos elementos destinados a estimular los sentidos y que es lo que Crawford (2003) denomina gratificación sensorial, En este ámbito, de los videojuegos, los componentes que constituyen los ambientes virtuales sustentados en las imágenes $3 \mathrm{D}$, la incorporación de sonidos cada vez más realistas y la interactividad que permiten, los convierten en ambientes emocionales por excelencia. Nos resulta, asombroso la evolución vertiginosa, no solo de los softwares, y los diseños, sino también de los elementos del hardware, que incorporan tarjetas de video y mecanismos de sonido tipo sound round, requeridos para la ejecución de los videojuegos, cuyo propósito no es otro que hacer más "vivencial" el juego y por tanto aumentar la sensación de inmersión lograda mediante ese aumento de estimulación sensorial.

La relación de los sentidos y las emociones se puede sustentar muy claramente en el modelo de Modelo Del Cerebro Triuno (ver Figura 1), presentado por los neurocientíficos, según el cual nuestro cerebro está constituido por tres partes neurofisiológica y funcionalmente diferenciadas. Estas partes son el sistema reptil o básico: primario evolutivamente, encargado de las funciones autónomas, de las respuestas motoras y automáticas, de los hábitos, y de las funciones más básicas del organismo. El sistema límbico: desde el punto de vista evolutivo, constituye el segundo cerebro, el cual se encarga de las emociones, las motivaciones, los sentimientos; controla el sistema autónomo del organismo. Trabaja en armonía con el sistema reptil, procesando toda la información sensorial antes de enviarla a la neocorteza. Y la Neocorteza o cerebro humano: evolutivamente es el cerebro más reciente, se encarga de la actividad intelectual, de los procesos superiores del hombre. Procesa información, genera conocimientos, imagina y anticipa, se encarga de la solución de problemas, de los procesos de este análisis y síntesis, del pensamiento crítico y creativo. Está formado por los dos hemisferios cerebrales: derecho e izquierdo, cada uno con su funciones específicas pero conectados a través de otra estructura neuronal que es el cuerpo calloso (Martínez, 2000).

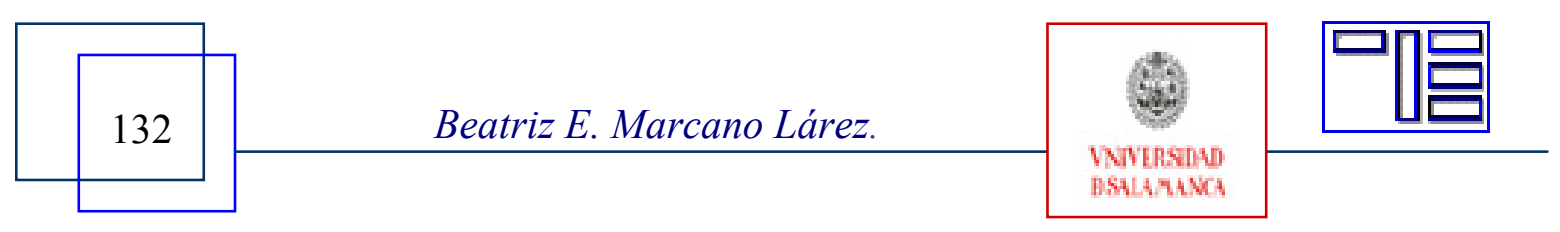




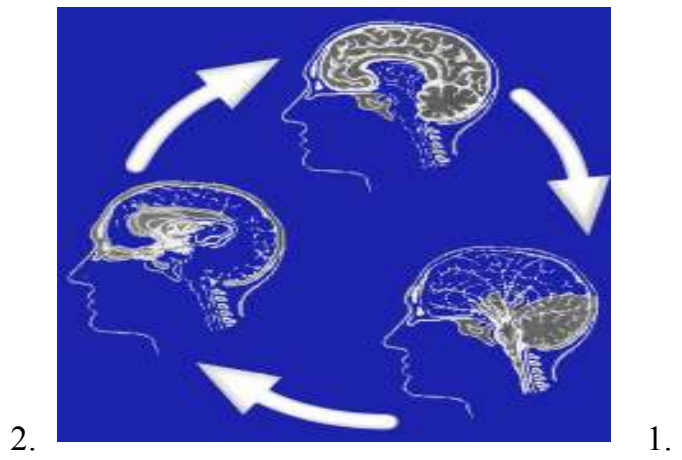

Figura 1. Modelo de Cerebro Triuno. Tomado de: Montes (1997)

A través de este modelo se puede visualizar muy claramente la conexión entre las vías aferentes y los centros de recepción de señales ubicadas en el sistema límbico, que constituye el centro emocional por excelencia del cerebro humano. En el sistema límbico se encuentran ubicadas la amígdala y el hipotálamo (Figura 2) responsables de las experiencias de las diferentes emociones y que están conectados con los órganos neurovegetativos (figura 3) que manifiestan estos estados emocionales con síntomas como aceleración del ritmo cardíaco, aumento del ritmo respiratorio, dilatación de las pupilas, sudoración, entre otros, los cuales son observables y medibles, tal como lo hicieron Shilling, Zyda y Wardynski (2004), para probar las respuestas emocionales ante el videojuego America's Army y otros juegos de simulación. Querían probar los efectos de sonido en la producción de respuestas emocionales a través de manifestaciones corporales como las mencionadas anteriormente, y encontraron una relación positiva entre los efectos de sonido que reforzaban el realismo en las situaciones de tensión y de peligro a las que virtualmente se enfrentaban los sujetos experimentales, en el caso de ellos, se trataba de estudiantes de la academia militar de las Fuerzas Armadas de los Estados Unidos.

Estas conexiones explícitas entre las vías sensoriales y el centro de las emociones, el sistema límbico, respaldan en buena medida los efectos emocionales que produce los videojuegos a través de la estimulación sensorial que ofrecen al usuario a través del diseño de sus interfaces.

A nuestro juicio los videojuegos constituyen una excelente herramienta de multiestimulación cognitivo afectiva que acelera el aprendizaje, genera placer, y potencia las habilidades digitales, el pensamiento estratégico y la creatividad, dependiendo en mayor o menor medida del tipo o género de videojuego que más se juegue. En este sentido, desde la óptica de la enseñanza, hay que aprovechar estas herramientas surgidas y desarrolladas desde un campo más comercial que didáctico, pero que ha echado sus anclas en una conducta básica de muchos mamíferos como es la conducta de jugar.

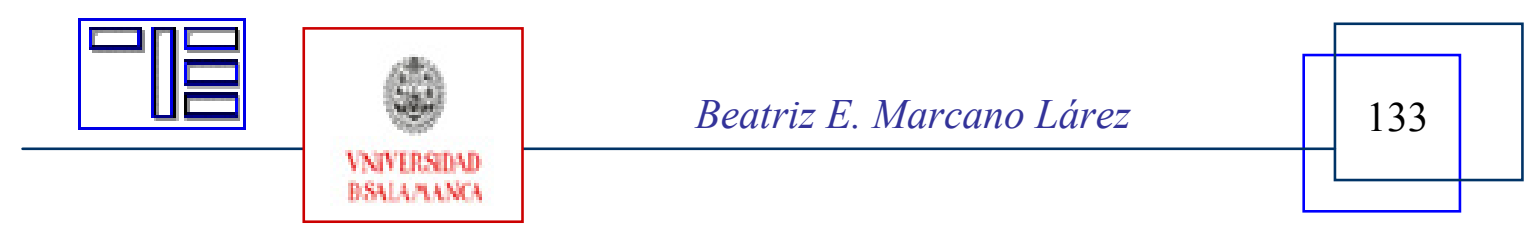


Revista Electrónica Teoría de la Educación.

Educación y Cultura en la Sociedad de la Información.

http://www.usal.es/teoriaeducacion

Vol. 7. No2. Diciembre 2006

Figura 2. Sitema Límbico. Tomado de Beauport $(1994,121)$
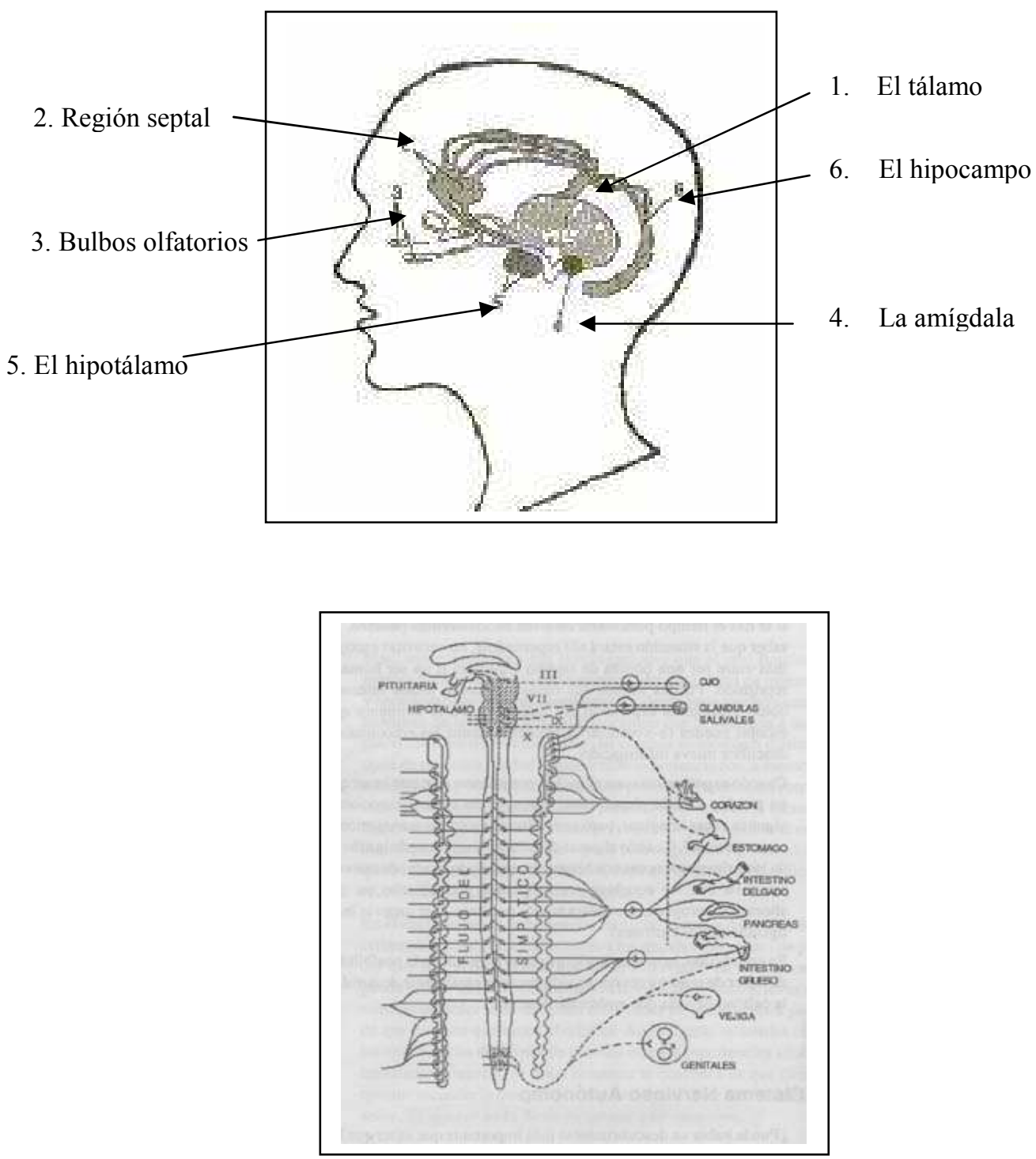

Figura 3 Diagrama simplificado del sistema nervioso autónomo y conexión con hipotálamo. Tomado de Beauport (1994, 136)

\section{3.- VIDEOJUEGOS Y APRENDIZAJE.}

Los videojuegos, además de ser una forma de ocio, podrían ser utilizados para aprender, si se les da uso didáctico. Sin embargo afirmamos, en concordancia con Crawford (1982), con los videojuegos se aprende independientemente de que éstos sean utilizados como estrategia didáctica. Como se indicó al referirnos al modelo de cerebro triuno, la exposición y la interacción con estos recursos de múltiple estimulación sensorial en la

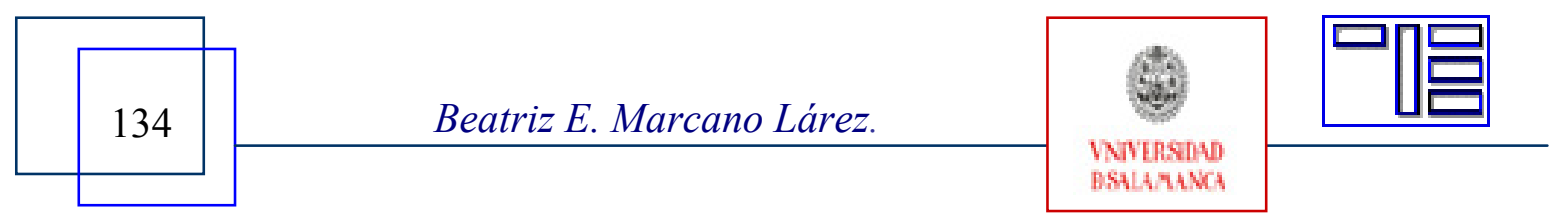


que están presentes elementos que afectan la estructuras encargadas de los estados emocionales de la personas y conociendo que estos estados afectivos a la vez afectan a la estructuras responsables de las cogniciones y el procesamiento de la información, de las relaciones entre las experiencias previas, y de los elementos almacenados en la memoria a largo y corto plazo, no cabría la menor duda del aumento en aprendizajes a medida que se practican con estas herramientas electrónicas.

Asimismo, resulta interesante analizar la habilidad de coordinación viso-motora que se requiere para operar exitosamente en los espacios virtuales que ofrecen los videojuegos. A mayor nivel de práctica más fácil es generalizar las respuestas de encadenamiento sináptico entre el teclear y la movilización en los espacios 3D, ya que esta práctica recurrente daría como resultado la formación de engramas que serían asumidas por el sistema básico o reptil del cerebro, encargado de las acciones y de las respuestas automáticas.

En cierta medida, basado en estos elementos puede considerarse los videojuegos como una forma efectiva de alfabetización digital, no sólo por la semiótica que se genera en torno a todo su funcionamiento, sino también por la forma de interactuar con los artefactos tecnológicos que invaden la vida cotidiana. (Gee, 2004, Gross, 2004; Levis, 2005). De esta manera, los videojuegos se convierten cada día más en una manera de comunicarse, de expresarse, y de transmitir las emociones y conocimientos que se tienen acerca de la realidad o de los mundos ficticios que se deseen crear.

Resulta interesante en este punto, responder a interrogantes relativas a los aprendizajes a nivel cognitivo, afectivo, y sociocultural que se obtiene a través de la ejecución de los videojuegos. Para responder a estos planteamientos se hará referencia a algunas investigaciones en el área.

\subsection{A nivel de senso-percepción:}

En algunas investigaciones acerca de los efectos de los videojuegos se ha encontrado que con su práctica se mejora la agudeza de percepción visual y la rapidez de reflejos (Raña, 2003; Pérez, 2005).

\subsection{A nivel cognitivo:}

El grupo F9 ha tomado la iniciativa de incorporar a sus prácticas docentes el uso de videojuegos como herramientas didácticas, obteniendo interesantes resultados en cuanto al desarrollo de las habilidades cognitivas y metacognitivas de los alumnos con los que han hecho sus pruebas. (Gros, 2004). Por su parte Raña (2003) cita diversas investigaciones en las que se indican que tanto los videojuegos como los microcyberjuegos (1) facilitan el desarrollo de estrategias más o menos complejas para la resolución de problemas, habiendo sido utilizados con éxito en las ciencias sociales.

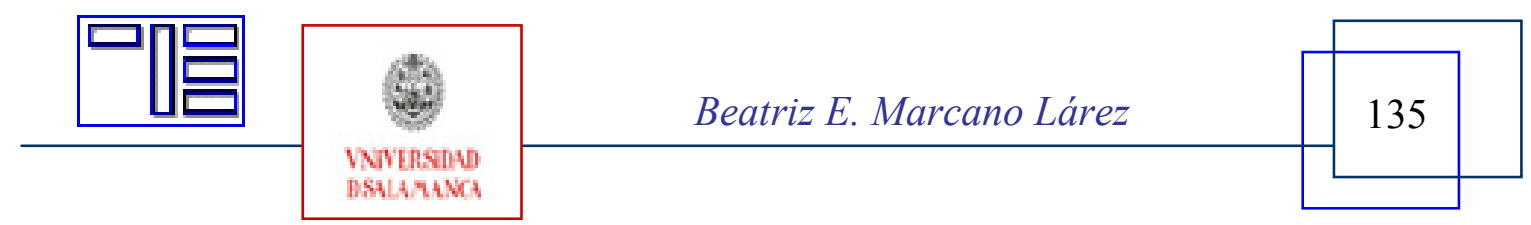


Revista Electrónica Teoría de la Educación.

Educación y Cultura en la Sociedad de la Información.

http://www.usal.es/teoriaeducacion

Vol. 7. No2. Diciembre 2006

En muchos casos no hay resultados concluyentes en cuando al aprendizaje de habilidades cognitivas, por cuanto la manera de aprender en el salón de clases y en los ambientes virtuales son muy diferentes, de allí la necesidad de profundizar en esta área de estudios Winn (2002).

\subsection{A nivel afectivo:}

Entre los efectos de los videojuegos también se encuentran los que repercuten en la autoestima, en la seguridad personal, en la motivación de logro y la capacidad de autosuperación, además de otras repercusiones a nivel de la atención, prerrequisito para cualquier proceso de aprendizaje.

En un interesante estudio realizado en la universidad de Madrid se aplicó un cuestionario a 2876 personas de todas las edades, hombres y mujeres, que incluía preguntas sobre cómo se siente el jugador durante y después de la partida, de los videojuegos, entre otros cuestionamientos, y se concluyó que los videojuegos potencian las habilidades como el liderazgo y la capacidad de autosuperación ya que más de la mitad de los menores de 35 años encuestados afirmaron haber mejorando sus capacidades de liderazgo y de superación (53,62\%) (Pérez, 2005).

En un estudio realizado sobre la agresividad se encontró que estos juegos tuvieron un efecto positivo en las niñas más tímidas e introvertidas, al ayudarlas a expresarse con más seguridad y asertividad. Por otra parte también se tienen evidencias del uso de los videojuegos en la recuperación de pacientes con enfermedades terminales ayudándoles a mejorar el ánimo y por tanto mejorando las condiciones del sistema inmune, pues como se señaló anteriormente la conexión existente entre el sistema límbico del cerebro, centro emocional por excelencia y el sistema neurovegetativo, puede explicar el efecto de los videojuegos en la salud a través de sus efectos en la producción de estados emocionales de placer y de logro (Michael y Chen, 2006).

Por otra parte se presentan hallazgos desde el área de la neuropsicología que relacionan la práctica de estos juegos con las zonas de recompensa y placer del cerebro (Anthuna, 2005), esto podría explicar lo atrapantes que resultan los juegos electrónicos, y hace reflexionar sobre el potencial que tienen como aceleradores del aprendizaje.

\subsection{A nivel sociocultural.}

Los videojuegos constituyen una excelente herramienta de alfabetización digital a través del cual se transmiten formas, valores, maneras de comportamiento, de relacionarse con otros, así como también - y esto no se había mencionado- los códigos y valores culturales y políticos predominantes en los países en los que se encuentran los mayores centros de producción y diseño de videojuegos, ubicados en los países desarrollados antes mencionados ejerciendo de esta manera su influencia en sus propios países y en aquellos

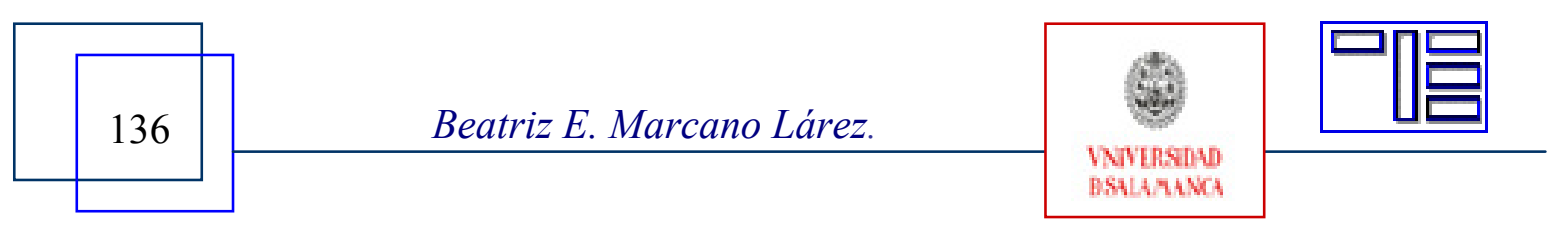


mayoritariamente "consumidores" de videojuegos (Espinoza, 2000; Gee, 2004; Díez,2006).

Por otra parte Woods (2003) diserta acerca de los juegos de simulación de situaciones sociales "serias" y afirma que los sistemas sociales simulados en los juegos combina roles, reglas y otros elementos del juego que realmente replican el sistema social existente permitiéndole al participante sin experiencia colocarse en una posición que le facilita una mayor comprensión de la dinámica de las fuerzas sociales que comparte la sociedad.

Gros (2000) considera que los videojuegos permiten aprender diferentes tipos de habilidades y estrategias. a) Ayudan a dinamizar las relaciones entre los niños del grupo, no sólo desde el punto de vista de la socialización sino también en la propia dinámica de aprendizaje. b) Permiten introducir el análisis de valores y conductas a partir de la reflexión de los contenidos de los propios juegos.

Otro efecto positivo de los videojuegos a nivel de sociabilidad es que gracias a la posibilidad de comunicación a través de la red los adolescentes comparten por esta vía con grupos de amigos y pares y no necesariamente permanecen aislados (Gros, 2004; Jansz y Martens ,2005; Pérez, 2005)

Por su parte Revuelta (2004), reflexiona sobre el juego en la red y los videojuegos, las implicaciones educativas y sociales que supone y la labor de la psicopedagogía en el nuevo campo de aprendizaje que genera la sociedad de la información. El autor destaca la necesidad de incorporar los videojuegos y los juegos online a las aulas y a los contenidos curriculares, por su alto potencial de socialización y de aprendizaje.

Hay que destacar en este punto que los efectos en los niveles afectivos y socioculturales están estrechamente ligados, por cuanto somos seres sociales, que vivimos la afectividad en relación con los otros, que sentimos placer, dolor, tristeza, alegría, frustración, en función del intercambio comunicacional que tengamos con los otros seres, sean ficticios, sean humanos o sus creaciones culturales, representadas en ideas o prejuicios a través de los personajes de los juegos, siempre y cuando éstos pertenezcan a los géneros de aventuras, de acción, de rol, o estrategia, es decir aquellos que requieran de un mayor procesamiento de información y que involucren mayores procesos cognitivo emocionales básicos y superiores (atención, concentración, análisis, toma de decisiones, resolución de problemas) y no con los juegos tipo Tetriz.

\section{4.- CONCLUSIONES}

Los videojuegos han constituido una gran fuente de entretenimiento en los últimos 20 años y una potente herramienta de aprendizaje. Para ello se han valido de una serie de elementos relativos al diseño gráfico, el diseño artístico, la ingeniería de sistemas, la programación, la ingeniería de sonido, entre otros. Así por ejemplo, los efectos de soni-

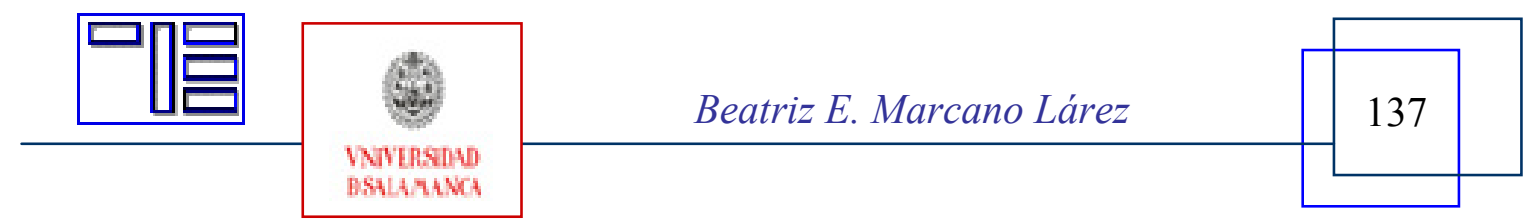


Revista Electrónica Teoría de la Educación.

Educación y Cultura en la Sociedad de la Información.

http://www.usal.es/teoriaeducacion

Vol. 7. No2. Diciembre 2006

do, la interactividad y la interfaz gráfica son importantes elementos para crear la sensación de inmersión, el disfrute y la gratificación sensorial que se tiene en la ejecución de buenos videojuegos.

Todos estos elementos convergen para actuar en las estructuras emocionales (sistema límbico) de los videojugadores favoreciendo diversos aprendizajes entre los que se pueden contar aquellos relacionados con conocimientos básicos sobre las interfaces digitales a niveles de llegar a automatizar las respuestas que permiten el éxito en la interacción con los elementos de los entornos virtuales diseñados para los videojuegos. Además de favorecer el aumento de la autoestima, la motivación al logro, el desarrollo de habilidades directivas y el trabajo en equipo, emplear Videojuegos, es una modalidad de enseñanza y entrenamiento que se esta aplicando actualmente en los campos de la salud, los servicios públicos, la educación y el área militar.

La cantidad de elementos emocionales que involucran los Videojuegos, a través de la estimulación sensorial y la posibilidad de inmersión a través de los ambientes virtuales en los que se desenvuelven y en los que hay que "meterse" para desenvolverse con éxito en la partida del juego, constituyen la principal fuente de atracción de estos softwares de entretenimiento, y como tales deben ser aprovechados por los entes educativos con más vigor, en virtud del gran potencial de aprendizaje que catapultan en diversas áreas tanto cognitivas-afectivas como practicas.

\section{5.- BIBLIOGRAFÍA}

ANTHUNA, E. (2005): Lo lúdico como promotor del bienestar de los niños en diversos contextos. Simposio. $30^{a}$ Congreso Interamericano de Psicología. Buenos Aires. Disponible en: http://www.eql.com.ar/sip2005/simposios/det_simposios.asp?simID=662 Consultado el 05-05-2005

CRAWFORD, C. (1982): The art of game desing. Disponible en:

http://www.vancouver.wsu.edu/fac/peabody/game-book/Coverpage.html. Consultado el 03-05-2006

DE BEAUPORT, E. (1994): Las tres caras de la mente. Caracas, Editorial Galac.

DÍEZ, E. (2006): Los videojuegos como mecanismos de transmisión educativa en las nuevas generaciones Didáctica, Innovación y Multimedia Grupo de investigación Didáctica y Multimedia de la UAB, 1 (4) Disponible en:

http://dewey.uab.es/pmarques/dim/revista.htm Consultado el 21/04/2006.

ESPINOZA, D. (2000): La tolerancia en los videojuegos: Una lectura con mapas axiológicos. Tesis Doctoral, Universidad de Alicante.

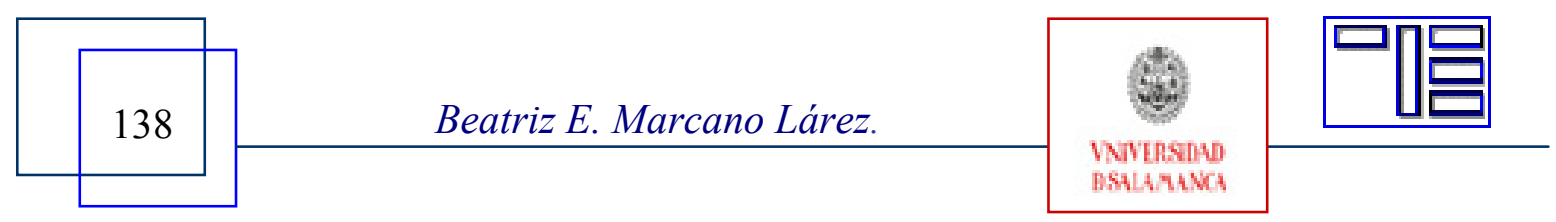


GEE, J. (2004): Lo que nos enseñan los videojuegos sobre el aprendizaje y el alfabetismo. Málaga, Aljibe.

GROS, B. (2000): La dimensión socioeducativa de los videojuegos. Revista Electrónica de Tecnología Educativa, 12.

GROS, B. (2004): Pantallas, Juegos y alfabetización. España, Desclée.De Brouwer. S.A.

JANZ, J. y MARTEN, L. (2005): Gaming at a LAN event: the social context of playing video games. New media \& society, 7 (3), 333-355. Disponible en: http://users.fmg.uva.nl/jjansz/janszmartens.pdf Consultado el 03-04-2006

LEVIS, D. (2005): Videojuegos y alfabetización digital. Aula de innovación educati$v a, 147$.

MARTÍNEZ, M. (2000): Revisión del Proceso Enseñanza- Aprendizaje a la luz de la Neurociencia (aprender con todo el cerebro). PERFILES, 24, 9-19. Disponible en: http://prof.usb.ve/miguelm. Consultado el 21-01-03.

MICHAEL, D. y CHEN, S. (2006): Serious Games. Games that educate, train and infoms. Canadá, Thonsom.

MONTES, Z. De (1997): Más allá de la educación. Caracas, Editorial Galac.

PÉREZ, J. (2005): Los videojuegos mejoran la sociabilidad y las "habilidades directivas. Disponible en:

http://www.cadenaser.com/articulo.html?xref=20051222csrcsrtec 2\&type=Tes Consultado el 23/12/2005.

RAÑA, J. (2003): Los microcyberjuegos y el aprendizaje de las ciencias sociales: el mundo Java. Relieve, 9:2, 236-246. Disponible en:

http://www.uv.es/RELIEVE/n9v2/RELIEVEv9n2 6.htm Consultado el 03-03-2005.

REVUELTA, F (2004): El poder educativo de los juegos on-line y de los videojuegos, un nuevo reto para la psicopedagogía en la sociedad de la información Theoria, 13, 97102.

SHILLING, R.; ZYDA, M. y WARDYNSKI, C. (2004): Introducing Emotion into Military Simulation and Videogame Design: America's Army: Operations and VIRTE. Disponible en: http://gamepipe.isi.edu/ zyda/pubs/ShillingGameon2002.pdf Consultado el 03-03-2006.

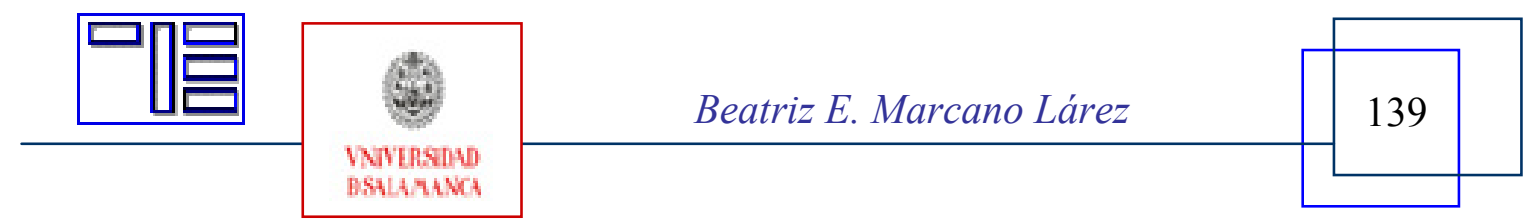


Revista Electrónica Teoría de la Educación.

Educación y Cultura en la Sociedad de la Información.

http://www.usal.es/teoriaeducacion

Vol. 7. No2. Diciembre 2006

WINN, W (2002): What can students learn in artificial environments that they cannot learn in class? University of Washington. Paper presented at the First International Symposium, Open Education Faculty, Anadolu Unversity, Turkey, May. Disponible en: http://faculty.washington.edu/billwinn/papers/turkey.pdf Consultado el 03-03-2006.

WOODS, S. (2003): Loading the Dice: The Challenge of Serious Videogames. The International Journal of Computer Game Research. 3, (2).

Disponible en: http://www.gamestudies.org/0401/woods/ Consultado el 25-04-2005)

\section{Notas}

[1] Microcyberjuegos: término con el que se agrupa el conjunto de juegos electrónicos de menor envergadura que los videojuegos que se encuentran disponibles por Internet en forma gratuita y son diseñados para ser accesibles on-line, por lo que no son excesivamente grandes, generalmente están desarrollados en java, javascrip y flash. (Raña, 2003).

\section{Para citar este artículo puede utilizar la siguiente referencia:}

MARCANO LÁREZ, Beatriz (2006): Estimulación emocional de los videojuegos: efectos en el aprendizaje. En GARCÍA CARRASCO, Joaquín (Coord.) Estudio de los comportamientos emocionales en la red [monográfico en línea]. Revista electrónica Teoría de la Educación: Educación y Cultura en la sociedad de la información. Vol. 7, $\mathrm{n}^{\circ}$ 2. Universidad de Salamanca. [Fecha de consulta: $\mathrm{dd} / \mathrm{mm} / \mathrm{aaaa}$ ].

$<$ http://www.usal.es/ teoriaeducacion/rev_numero_07_02/n7_02_beatriz_marcano.pdf $>$ ISSN 1138-9737

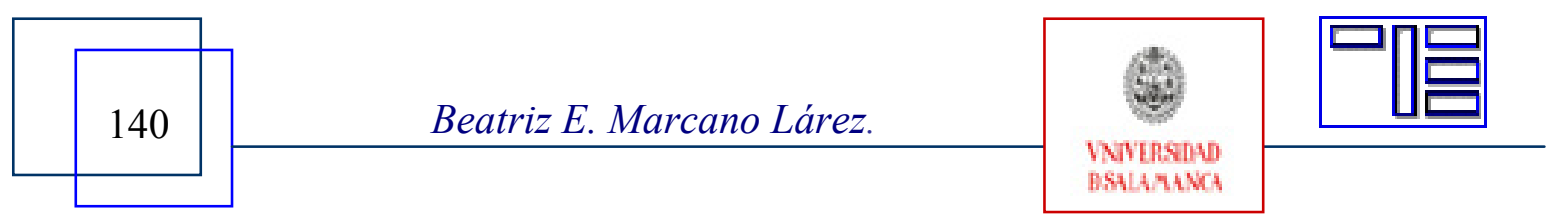

